Article

\title{
New Advances in the Determination of Free and Bound Phenolic Compounds of Banana Passion Fruit Pulp (Passiflora tripartita, var. Mollissima (Kunth) L.H. Bailey) and Their In Vitro Antioxidant and Hypoglycemic Capacities
}

\author{
Elisa Giambanelli ${ }^{1}$, Ana Maria Gómez-Caravaca ${ }^{1, *}$, Arancha Ruiz-Torralba ${ }^{2}$, \\ Eduardo Jesús Guerra-Hernández ${ }^{2} \mathbb{D}$, Jorge Geovanny Figueroa-Hurtado ${ }^{3}{ }^{(D}$, \\ Belén García-Villanova ${ }^{2}$ (D) and Vito Verardo $2,4, * \mathbb{D}$ \\ 1 Department of Analytical Chemistry, Faculty of Sciences, University of Granada, Avd. Fuentenueva s/n, \\ 18071 Granada, Spain; elisagiambanelli@ugr.es \\ 2 Department of Nutrition and Food Science, University of Granada, Campus of Cartuja, 18071 Granada, \\ Spain; ruizarancha@ugr.es (A.R.-T.); ejguerra@ugr.es (E.J.G.-H.); belenv@ugr.es (B.G.-V.); \\ 3 Departamento de Química y Ciencias Exactas, Universidad Técnica Particular de Loja, San Cayetano Alto \\ s/n, 11-01-608 Loja, Ecuador; jgfigueroa@utpl.edu.ec \\ 4 Institute of Nutrition and Food Technology 'José Mataix', Biomedical Research Center, University of \\ Granada, Avda del Conocimiento sn., 18100 Armilla, Granada, Spain \\ * Correspondence: anagomez@ugr.es (A.M.G.-C.); vitoverardo@ugr.es (V.V.); Tel.: +34-958-243-339 \\ (A.M.G.-C.); +34-958-243-863 (V.V.)
}

Received: 23 June 2020; Accepted: 14 July 2020; Published: 17 July 2020

\begin{abstract}
Banana passion fruit (Passiflora tripartite L.H. Bailey) is a lesser known species of the genus Passiflora. This fruit typically grows in the Andean region of Ecuador and it is locally known as tumbo, taxo or curuba. The juice of this fruit is highly appreciated in South America. Extracts of banana passion fruit were characterized for their content levels of free and bound phenolic compounds by high performance liquid chromatography coupled to high resolution mass spectrometry detector (HPLC-ESI-TOF-MS). A total of 82 polar compounds classified as phenolic acid derivatives, organic acids, benzophenones, flavan-3-ols, flavonols and flavones were detected in the extracts. The total phenolic content was $2356 \mathrm{mg} 100 \mathrm{~g}^{-1}$ dry matter, with the bound phenolic fraction representing $37.7 \%$ of total amounts. Flavan-3-ols, such as (epi)catechin, (epi)azfelechin and their derivatives, were the main phenolic compounds in the free phenolic fraction; however, phenolic acids represented the most abundant class of bound phenolic extracts. The antioxidant and hypoglycemic capacities reported for banana passion fruit were higher than for other fruits. To our knowledge, this is the first time that bound phenolic compounds have been described in banana passion fruit pulp.
\end{abstract}

Keywords: fruit; Passiflora; bioactive compounds; HPLC-ESI-TOF-MS; flavan-3-ols; proanthocyanidins; DPPH; ABTS; FRAP; antihyperglycemic activity

\section{Introduction}

Tropical fruits, with their delicious taste, refreshing flavor and sweetness, are particularly appreciated by consumers. They are often processed into juices, puree, canned fruits, jam and dehydrated bars. In the last few decades, their popularity has increased around the world, especially in developed countries, because they are associated with health promoting compounds [1-3]. 
Several investigations carried out in the last few years have highlighted the fact that these fruits are important sources of antioxidants such as phenolic compounds, vitamins, carotenoids and minerals [1-23]. Their high phytochemical content contributes to their preventive effect against chronic degenerative diseases.

Latin America hosts a high number of crops and substantial tropical fruit biodiversity; the genetic resources of this area, still poorly exploited, could be addressed in order to deal with challenges such as sustainable agriculture development and food security [4]. The Andean region of Ecuador has a wealth of underexploited native and exotic fruits, for which the local population still maintains traditional uses [5,6]. The genus Passiflora, comprising about 500 species, is the largest genus in the family of Passifloraceae [7]. The leaves of this genus are mainly used and exploited for their phytotherapeutic properties as anti-inflammatory, anxiolytic and sedative substances [8,9]. Within this genus, the passion fruit varieties of Passiflora edulis Sims F. flavicarpa Degener and Passiflora edulis Sims F. edulis, most commonly known as maracuyá and gulupa, are highly appreciated by consumers [10]. A lesser known species of the genus Passiflora is banana passion fruit (Passiflora tripartita var. Mollissima L.H. Bailey). This fruit typically grows in the Andean region, and it is locally known as tumbo, curuba or taxo. With the typical shape of a banana, the fruit can reach around $40-110 \mathrm{~g}$ in weight and $8-15 \mathrm{~cm}$ in length. The pulp is gelatinous, and it surrounds several small black seeds. Locally, it is mainly consumed as juice, and its unique taste could be of particular interest to be exploited in niche markets.

Recently, leaves, pulp, seeds and edible parts of banana passion fruit have been investigated regarding their composition, especially their phytochemical content [11-13]. Previous studies demonstrated that banana passion fruit is particularly rich in phenolic antioxidants [14,15]. Contreras-Calderón et al. [16] found that banana passion fruits had the highest levels of antioxidant activity compared to other fruits, and this fact has been attributed to the presence of high amounts of phenolic compounds [12,13]. As a result, there are studies that have investigated the evolution of these compounds following the microencapsulation of the fruit's pulp [15].

The presence of phenolic compounds in foods is commonly investigated to determine their putative protective effects against several chronic diseases, as also highlighted by several experimental studies [17-19]. Phenolics in fruits and vegetables occur as soluble conjugated and insoluble forms, covalently bound to sugar moieties or to cell wall structures. These forms have a different absorption pathway in the digestive tract, with bound phenolic compounds reaching the colon intact in order to fully release and exhibit their bioactivity [20]. Several studies have also demonstrated the significantly higher antioxidant capacity of the insoluble bound phenolics with respect to free and soluble conjugated phenolics [21]. Notwithstanding this, investigations carried out on the phenolic content of fruits are mainly addressed at characterizing free phenolic compounds, underestimating the extent to which the total phenolic content is the sum of free and bound phenolic compounds.

To our knowledge, banana passion fruit has never been analyzed and characterized for its content of bound phenolic compounds before. Consequently, the main aim of this study was the identification and quantification of free and bound phenolic compounds in banana passion fruit pulp by high performance liquid chromatography coupled to high resolution mass spectrometry detector (HPLC-ESI-TOF-MS). Moreover, the fruit's antioxidant and hypoglycemic capacities were also assayed.

\section{Materials and Methods}

\subsection{Chemicals}

HPLC-grade acetonitrile, methanol, acetic acid, ethanol, hexane, ethyl acetate, diethyl ether and hydrochloric acid were purchased from Merck KGaA (Darmstadt, Germany). Hydroxide sodium was obtained from Fluka (Buchs, Switzerland). Double-deionized water with conductivity lower than 18.2 M $\Omega$ was obtained with a Milli-Q system from Millipore (Bedford, MA, USA). Ferulic acid, catechin, quercetin and rutin (Sigma-Aldrich, St. Louis, MO, USA) were used for the calibration curves. 


\subsection{Sample}

About $5 \mathrm{~kg}$ of Passiflora tripartita var. Mollissima fruits were purchased from a local market in Loja (Ecuador). Pulp was manually separated, immediately frozen in encoded plastic bags at $-20^{\circ} \mathrm{C}$, and then freeze-dried (Thermo HETO, powerdry LYOLAB 3000; Waltham, MA, USA). Dried samples were ground to a fine powder in a blender mixer (Ika-Werke M20; Staufen, Germany) and stored at $-18^{\circ} \mathrm{C}$ until extraction.

Before the drying process, soluble solids ( ${ }^{\circ}$ Brix $), \mathrm{pH}$ and acidity were determined, and the results are shown in Table 1.

Table 1. Soluble solids ( ${ }^{\circ}$ Brix), $\mathrm{pH}$ and acidity values.

\begin{tabular}{ccc}
\hline Parameter & Result & Reference Method [22] \\
\hline Soluble solids ( ${ }^{\circ}$ Brix $)$ & $11.42 \pm 0.03$ & AOAC 932.12 \\
pH & $3.53 \pm 0.02$ & AOAC 981.12 \\
Acidity (g/100 g of citric acid equiv.) & $1.08 \pm 0.02$ & AOAC 942.15 \\
\hline
\end{tabular}

\subsection{Free and Bound Phenolic Compound Extraction}

The free phenolic fraction of pulp was extracted with the protocol suggested by Gómez-Caravaca et al. [23]. Briefly, $0.5 \mathrm{~g}$ of the previously freeze-dried sample was extracted using $10 \mathrm{~mL}$ of ethanol/water $(80: 20, v / v)$, using an ultrasonic bath (15 min at room temperature). After this, the resulting sample was centrifuged $(15 \mathrm{~min}$ at $1000 \mathrm{~g})$. This extraction process was replicated twice more. The solvents of the three extractions were pooled, carried out to dryness in a rotavapor and re-dissolved in $3 \mathrm{~mL}$ of methanol/water $(50: 50, v / v)$. The bound polar fraction was obtained after alkaline hydrolysis of the remains from free phenolic extraction. Digestion of the samples was performed at room temperature with $\mathrm{NaOH} 2 \mathrm{M}(100 \mathrm{~mL})(20 \mathrm{~h}$ under nitrogen atmosphere). Then, $\mathrm{HCl}$ was used to reach $\mathrm{pH}$ 2-3 in a cooling ice bath, and $500 \mathrm{~mL}$ of hexane was added to eliminate the lipid fraction. Three extractions with $100 \mathrm{~mL}$ of diethyl ether/ethyl acetate $(50 / 50 \mathrm{v} / \mathrm{v})$ were conducted; the fractions were collected and evaporated to dryness. The obtained extract was re-dissolved in $2 \mathrm{~mL}$ of methanol/water $(1: 1 \mathrm{v} / \mathrm{v})$.

Finally, $0.22 \mu \mathrm{m}$ PTFE syringe filters were used to filter the extracts, and they were kept at $-18{ }^{\circ} \mathrm{C}$.

\subsection{HPLC-ESI-TOF-MS Analyses}

Free and bound phenolic compounds were determined using an ACQUITY UPLC M-Class liquid chromatograph from Waters (Waters Corp., Milford, MA, USA) that included a degasser, a binary pump, an oven and an automatic liquid sampler, and it was coupled with mass spectrometry. Phenolic compounds were separated on HPLC column Poroshell 120, SB-C18 (3.0 mm × $100 \mathrm{~mm}$, $2.7 \mu \mathrm{m}$ ) from Agilent Technologies (Agilent Technologies, Palo Alto, CA, USA). Oven temperature was set at $25^{\circ} \mathrm{C}$ and separation was conducted according to the conditions previously proposed by Gómez-Caravaca et al. [23].

\subsection{Evaluation of Antioxidant Capacity}

The antioxidant capacity of the extracts was evaluated using three assays, namely DPPH, ABTS and FRAP. The analyses were developed on Fluostart Omega microplate apparatus (BMG LabTech, $\mathrm{GmbH}$, Ortenberg, Germany). The protocols used were the same adopted by Ruiz-Torralba et al. [24]. The results are the mean of three replications and are expressed as $\mu$ mol Trolox equivalent (TE)/g of sample dry weight (d.w.).

\subsection{In Vitro Hypolycemic Capacity via $\alpha$-Amylase Inhibitory Assay}

The $\alpha$-amylase inhibitory test was carried out according to Leporini et al. [25], measuring the production of maltose after the incubation of starch with $\alpha$-amylase (with and without Passiflora passion 
fruit extract). The extract was added to 3,5-dinitrosalicylic acid and the reaction mixture was measured at $540 \mathrm{~nm}$.

\subsection{Statistical Analysis}

The results reported in this study are the averages of three repetitions $(n=3)$. Pearson's linear correlations at the $p<0.05$ level were evaluated using Statistica 6.0 software (2001, StatSoft, Tulsa, OK, USA).

\section{Results}

\subsection{Identification of Free and Bound Phenolic Compounds}

Banana passion fruit is a popular fruit from Ecuador, especially appreciated for its juice. Free (FPC) and bound (BPC) phenolic compounds have been extracted from the pulp of this fruit and characterized by means of HPLC-ESI-TOF-MS (Figure S1). Extract ion chromatograms of the peaks, exact mass $(m / z)$, molecular formula and fragment ions formed at ion source, together with retention time, Error, mSigma and literature, have been taken into account for identification purposes.

A total of 82 peaks have been detected and tentatively identified in banana passion fruit pulp extracts. Identified compounds belonged to organic acids, benzophenones and different classes of phenolic compounds; among these were flavan-3-ols, flavonols and flavones derivatives, phenolic acids and derivatives. All these compounds are reported in Table 2, together with the main information obtained by the HPLC-ESI-TOF-MS platform.

Peaks 1 and 2, with molecular formulas $\mathrm{C}_{7} \mathrm{H}_{12} \mathrm{O}_{6}$ and $\mathrm{C}_{6} \mathrm{H}_{8} \mathrm{O}_{7}$, were identified as organic acids, specifically quinic acid and citric acid, respectively. These compounds are commonly present in numerous fruits [23].

Several peaks have been identified as phenolic acids and derivatives, especially in the first part of the chromatogram. Peaks 3, 4, 6 and 7 showed the same fragment ion at $\mathrm{m} / \mathrm{z} 191$ that is due to the presence of quinic acid deprotonated moiety. In particular, peaks 3, 4 and 6 showed the same molecular formula, $\mathrm{C}_{22} \mathrm{H}_{28} \mathrm{O}_{14}$, and they were assigned to three derivatives of dicaffeoylquinic acid. The presence of dicaffeoylquinic acids and caffeoylglucaric acid derivatives (peak 79, with $\mathrm{m} / \mathrm{z} 563.3261$ and molecular formula $\mathrm{C}_{24} \mathrm{H}_{52} \mathrm{O}_{14}$ ) was already confirmed in passion fruit juice by Spínola et al. [26]. Peak 7, with $\mathrm{m} / \mathrm{z} 527.1050$ and molecular formula $\mathrm{C}_{22} \mathrm{H}_{24} \mathrm{O}_{15}$, was assigned to a caffeoyltartaric acid derivative, according to Spínola et al. [26], who isolated the same molecule in cherimoyas. Another hydroxycinnamic acid derivative was peak 63 , which showed molecular formula $\mathrm{C}_{18} \mathrm{H}_{16} \mathrm{O}_{8}$ and $\mathrm{m} / \mathrm{z} 359.0895$ and which was tentatively identified as rosmarinic acid. This compound is a typical hydroxycinnamoyl derivative of Labiatae plants [27], but rosmarinic acid has also been isolated as a major compound, together with 3-O-caffeoylquinic acid, in Solanum betaceum Cav. cultivars from Ecuador [28]. Finally, peak 31 was identified as caffeic acid [11].

Among hydroxybenzoic acids, peaks 9 and 17 were respectively identified as 3,4-dihydroxybenzoic acid and 4-hydroxybenzoic acid. These phenolic acids have been recently found in the extracts of Passiflora mollissima seeds obtained by a pressurized liquid system and determined by UHPLC-Q-TOF-MS/MS analysis [11]. Peaks 10 and 23 were attributed to syringic acid, whereas peaks 69 and 81 were tentatively identified as syringic acid derivatives, specifically syringic acid hexoside and methylsyringin, respectively. Derivatives of syringic acid were previously detected in pineapple and mango by Septembre-Malaterre et al. [29]. Peaks 45 and 46, with molecular formula $\mathrm{C}_{20} \mathrm{H}_{20} \mathrm{O}_{14}$, $m / z 483.1093$ and a fragment ion at m/z 321.0748 due to the loss of a glucose moiety (M-162), were identified as two isomers of digalloylglucose; the same compounds were already identified by Gómez-Caravaca et al. [23] in mango fruits.

Besides phenolic acids, a large number of flavonoids was also detected in banana passion fruit pulp extracts. Flavones and flavonols were mainly present as hexoside derivatives. Among these, peak 16, with molecular formula $\mathrm{C}_{21} \mathrm{H}_{20} \mathrm{O}_{13}$ and $\mathrm{m} / \mathrm{z} 479.0881$, was attributed to the flavonol myricetin 
hexoside, which has already been described in guava leaves [30]. Another myricetin derivative (peak 80) was tentatively identified, with molecular formula $\mathrm{C}_{30} \mathrm{H}_{60} \mathrm{O}_{20}$ and $m / z$ 739.3630. This flavonol derivative was previously reported by Guimarães et al. [31] in wild fruits of Prunus spinosa from Northeastern Portugal. Peak 28, with molecular formula $\mathrm{C}_{12} \mathrm{H}_{20} \mathrm{O}_{12}$ and $m / z$ 463.0841, was attributed to isoquercitrin, a glucoside of quercetin that has been isolated in other tropical fruits, such as mango and guava leaves [23,30]. Peak 62, with molecular formula $\mathrm{C}_{28} \mathrm{H}_{32} \mathrm{O}_{17}$ and $\mathrm{m} / z$ 639.1273, was assigned to isorhamnetin-O-dihexoside, in accordance with Spínola et al. [26], who determined for the first time the conjugate form of this flavonol in passion fruit and strawberry.

Peak 50, with molecular formula $\mathrm{C}_{15} \mathrm{H}_{12} \mathrm{O}_{6}$ and $\mathrm{m} / \mathrm{z} 287.0666$, was identified as the flavanone eriodictyol, in agreement with previous HPLC-mass spectrometry screening of mango and its by-products [23]. Peaks 35 and 47, with molecular formula $\mathrm{C}_{21} \mathrm{H}_{22} \mathrm{O}_{11}$ and $m / z 449.1234$ and 449.1235, respectively, were tentatively assigned to isomeric forms of eriodictyol-O-hexoside, according to Pereira et al. [32]. Peak 70 was tentatively identified as phloretin dihexoside, showing a molecular formula of $\mathrm{C}_{27} \mathrm{H}_{33} \mathrm{O}_{15}$ and $m / z$ 597.2018. These compounds have mainly been described in tomato samples [33]. Peak 74, with molecular formula $\mathrm{C}_{30} \mathrm{H}_{26} \mathrm{O}_{10}$ and $m / z$ 545.1626, was also observed in extracts of Passiflora mollissima seeds by Ballesteros-Vivas et al. [11], and it was tentatively attributed to a trihydroxyflavanone dimer.

Peak 36 showed a molecular formula $\mathrm{C}_{21} \mathrm{H}_{20} \mathrm{O}_{11}$ and $m / z 447.0978$, and it was identified as luteolin-6-C-glucoside (orientin). This flavone has already been reported in passion fruit of the genus Passiflora edulis by Spínola et al. [26].

Peaks 73 and 77, with molecular formula $\mathrm{C}_{28} \mathrm{H}_{32} \mathrm{O}_{15}$ and $m / z$ 607.1625 and 607.1647, respectively, were identified as diosmetin-rutinoside. Peaks 43 and 54, with molecular formula $\mathrm{C}_{28} \mathrm{H}_{32} \mathrm{O}_{16}$ and $m / z$ 623.1646 and 623.1641, respectively, were identified as diosmetin-dihexoside (lucenin-2,4-methyl ether). Diosmetin-hexoside derivatives have been reported in citrus fruits [26], and diosmetin-rutinoside has been detected in peel samples of Passiflora mollissima [34].

Peak 51, with molecular formula $\mathrm{C}_{21} \mathrm{H}_{20} \mathrm{O}_{9}$ and $m / z$ 415.1068, was tentatively identified as daidzin. According to the literature, peak 76, with molecular formula $\mathrm{C}_{22} \mathrm{H}_{20} \mathrm{O}_{9}$ and $m / z 427.1048$, was assigned to durantin A; this compound was previously isolated and characterized by NMR in the whole plant of Duranta repens Linn, a subtropical species that is found in West Indies and South America [35]. Peak 56 showed a molecular formula of $\mathrm{C}_{21} \mathrm{H}_{18} \mathrm{O}_{11}$ and $m / z$ 445.0916; a similar peak was identified as baicalin by Li et al. [36] in Scutellaria lateriflora, a plant that is popular for its mild relaxant properties and is used as a therapy for anxiety, nervous tension and convulsions.

Peak 78, at $m / z 557.1256$ and molecular formula $\mathrm{C}_{27} \mathrm{H}_{26} \mathrm{O}_{13}$, was assigned to 2,6-dihydroxy -3-methyl-4-O-(6"-O-galloyl- $\beta$-D-glucopyranosyl)-benzophenone. This compound has been previously isolated in Psidium guajava L. fruits and leaves [30,37].

Flavan-3-ols represented a numerous class of phenolics in banana passion fruit pulp extracts. Peak 27, with molecular formula $\mathrm{C}_{15} \mathrm{H}_{14} \mathrm{O}_{6}$ and $\mathrm{m} / \mathrm{z} 289.0721$, was assigned to catechin, and its identification was also confirmed by co-elution with a commercial standard. Several peaks of the chromatogram showed a fragment ion at $m / z 289$, meaning that they were (epi)-catechin derivatives. Peaks 37 and 38, at $m / z$ 435.1230, showed a loss of 146 uma (M-146), producing a fragment ion at $m / z$ 289.1005, due to the loss of catechin monomer unit, and they were identified as catechin deoxyhexose isomers, according to de Souza Mesquita et al. [38], who found the same compound in the mangrove tree. Peaks 19, 29 and 30, with molecular formula $\mathrm{C}_{21} \mathrm{H}_{24} \mathrm{O}_{11}$ and $m / z$ 451.1262, showed a fragment ion at $m / z 289.0896$, and they were attributed to catechin glucoside isomers. A molecular ion at $m / z 451$ was also observed by García-Ruiz et al. [15] in freeze-dried and microencapsulated banana passion fruits; the major MS/MS fragment observed at $m / z 289$ indicated the loss of a hexose from the catechin moiety. Peaks 5, 11, 12, 13, 14 and 26 showed the same molecular formula, $\mathrm{C}_{15} \mathrm{H}_{14} \mathrm{O}_{7}$, with $\mathrm{m} / \mathrm{z}$ 305.2595, and they were attributed to (epi)gallocatechin isomers. Peaks 42,58 and 59 , with molecular formula $\mathrm{C}_{15} \mathrm{H}_{14} \mathrm{O}_{5}$ and $m / z 273$, were attributed to (epi)fisetinidol isomers, according to Ballesteros-Vivas et al. [11]. Peaks 48, 52, 53, 64, 65 and 68 showed molecular formula $\mathrm{C}_{21} \mathrm{H}_{24} \mathrm{O}_{10}$ 
and $m / z$ 435, with a characteristic fragment ion at $m / z 273$, corresponding to the glucosidic form of (epi)afzelechin, as also reported by García-Ruiz et al. [15]. Peaks 8, 25, 44 and 61, with molecular formula $\mathrm{C}_{27} \mathrm{H}_{30} \mathrm{O}_{16}$ and $m / z 609$, showed two fragment ions at $m / z 435$ and 273 , and they were identified as (epi)azfelechin glucoside derivatives, as reported by García-Ruiz et al. [15]. Peak 18, at $m / z 329.0892$ and molecular formula $\mathrm{C}_{17} \mathrm{H}_{14} \mathrm{O}_{7}$, was tentatively identified as kaempferol-methoxy-methyl ether, as already described by Song et al. in another Passiflora fruit [39]. Proanthocyanidins consist of oligomeric forms sharing the flavan-3-ol monomers (epi)catechin, (epi)gallocatechin, (epi)azfelechin or (epi)fisetinidol. Peaks 15, 21, 32, 39, and 40, with molecular formula $\mathrm{C}_{30} \mathrm{H}_{26} \mathrm{O}_{13}$ and $\mathrm{m} / \mathrm{z} 593$, were identified as (epi)gallocatechin-(epi)catechin isomers; several isomers of these compounds have also been found by Ballesteros-Vivas et al. [11] in banana passion fruit seeds. As expected, all these peaks showed the presence of a fragment at $\mathrm{m} / \mathrm{z} 289$, corresponding to the catechin monomer. Peaks 20 and 75 , with molecular formula $\mathrm{C}_{30} \mathrm{H}_{24} \mathrm{O}_{12}$ and $m / z 575$, were assigned to the A type procyanidin dimer (epi)catechin-(epi)catechin. Besides this, peaks 24,33 and 60, with molecular formula $\mathrm{C}_{30} \mathrm{H}_{26} \mathrm{O}_{12}$ and $\mathrm{m} / z$ 577 , were attributed to the $\mathrm{B}$ type procyanidin dimer (epi)catechin-(epi)catechin, as previously reported by Ballesteros-Vivas et al. [11]. The flavan-3-ol subunits of B type procyanidins are connected by a single bond, whereas in A type procyanidins, an additional ether linkage between adjacent monomers occurs. Peaks $34,49,55,57,66,67$ and 71 , at $m / z 561$ and molecular formula $\mathrm{C}_{30} \mathrm{H}_{26} \mathrm{O}_{11}$, were tentatively identified as trihydroxy(iso)flavanol-(epi)catechin isomers (B type linkage). García-Ruiz et al. [15] and Verardo et al. [40] observed this compound in banana passion fruit and buckwheat, respectively; the authors assigned these peaks to a propelargonidin dimer composed of (epi)azfelechin-(epi)catechin. The same molecular ion at $m / z 561$ was identified as (epi)fisetinidol-(epi)catechin by Ballesteros-Vivas et al. [11]. This choice was explained by the presence of the fragment ion at $m / z 409$ (with a loss M-152), due to the typical retro-Diels-Alder (RDA) reaction of flavonoids [41]. Peak 72, with molecular formula $\mathrm{C}_{45} \mathrm{H}_{38} \mathrm{O}_{16}$ and $m / z$ 833.2046, was assigned to the trimer (epi)azfelechin-(epi)azfelechin-(epi)catechin by Verardo et al. [40], whereas it was identified as (epi)fisetinidol-(epi)fisetinidol-(epi)catechin by Ballesteros-Vivas et al. [11]. Finally, another procyanidin trimer was attributed to peak 22, according to the findings of García-Ruiz et al. [15].

The peaks observed in the last part of the chromatogram (minutes 18-20) were tentatively identified as glucopyranoside, galactopyranoside and mannopyranoside derivatives; moreover, lactones and fatty acids were also observed. However, this was not the aim of the present study, so they will not be discussed in this paper. 
Table 2. Phenolic compounds identified as free and bound forms in banana passion fruit pulp.

\begin{tabular}{|c|c|c|c|c|c|c|c|c|}
\hline Peak N & Compound & RT (min) & Molecular Formula & Calculated Mass $[\mathrm{M}-\mathrm{H}]^{-}$ & Experimental Mass $[\mathrm{M}-\mathrm{H}]^{-}$ & Error (ppm) & mSigma & In Source Fragments \\
\hline 1 & quinic acid & 0.79 & $\mathrm{C}_{7} \mathrm{H}_{12} \mathrm{O}_{6}$ & 191.0197 & 191.0268 & 8.8 & 14.2 & \\
\hline 2 & citric acid & 0.94 & $\mathrm{C}_{6} \mathrm{H}_{8} \mathrm{O}_{7}$ & 191.0197 & 191.027 & 8.2 & 12.4 & \\
\hline 3 & dicaffeoylquinic acid derivative1 & 1.48 & $\mathrm{C}_{22} \mathrm{H}_{28} \mathrm{O}_{14}$ & 515.1406 & 515.1413 & 8.1 & 18.6 & $353.0976,191.0267,179.0674$ \\
\hline 4 & dicaffeoylquinic acid derivative 2 & 1.86 & $\mathrm{C}_{22} \mathrm{H}_{28} \mathrm{O}_{14}$ & 515.1406 & 515.1413 & -1.2 & 19.4 & $353.0976,191.0267,179.0674$ \\
\hline 5 & (epi)gallocatechin & 2.01 & $\mathrm{C}_{15} \mathrm{H}_{14} \mathrm{O}_{7}$ & 305.0667 & 305.0671 & -4.1 & 24.1 & 191.0294 \\
\hline 6 & dicaffeoylquinic acid derivative 3 & 2.15 & $\mathrm{C}_{22} \mathrm{H}_{28} \mathrm{O}_{14}$ & 515.1406 & 515.141 & -0.3 & 24.5 & 353.0976, 191.0271, 179.0503 \\
\hline 7 & caffeoyltartaric acid derivative & 2.38 & $\mathrm{C}_{22} \mathrm{H}_{24} \mathrm{O}_{15}$ & 527.1042 & 527.105 & -1.5 & 15.2 & $469.0998,349.0535,191.0285$ \\
\hline 8 & (epi)-azfelechin glucoside derivative & 2.95 & $\mathrm{C}_{27} \mathrm{H}_{30} \mathrm{O}_{16}$ & 609.1402 & 609.1442 & 2.6 & 9.1 & $527.1062,353.0854,191.0272$ \\
\hline 9 & 3,4-dihydroxybenzoic acid (protocatechuic) & 2.98 & $\mathrm{C}_{7} \mathrm{H}_{6} \mathrm{O}_{4}$ & 153.0193 & 153.0152 & -3.6 & 13 & \\
\hline 10 & syringic acid & 3.55 & $\mathrm{C}_{9} \mathrm{H}_{10} \mathrm{O}_{5}$ & 197.0455 & 197.0415 & -5.1 & 40.2 & $179.0342,167.0405,123.0528$ \\
\hline 11 & (epi)gallocatechin & 4.14 & $\mathrm{C}_{15} \mathrm{H}_{14} \mathrm{O}_{7}$ & 305.0667 & 305.0669 & -1.5 & 13.9 & - \\
\hline 12 & (epi)gallocatechin & 4.32 & $\mathrm{C}_{15} \mathrm{H}_{14} \mathrm{O}_{7}$ & 305.0667 & 305.0777 & -3.0 & 16.4 & $219.1106,191.0266$ \\
\hline 13 & (epi)gallocatechin & 4.37 & $\begin{array}{l}1511407 \\
\mathrm{C}_{15} \mathrm{H}_{14} \mathrm{O}_{7}\end{array}$ & 305.0667 & 305.0773 & -3.0 & 16.9 & $219.0607,191.0271$ \\
\hline 14 & (epi)gallocatechin & 4.48 & $\mathrm{C}_{15} \mathrm{H}_{14} \mathrm{O}_{7}$ & 305.0667 & 305.0773 & -2.9 & 15.8 & $219.0607,191.0271$ \\
\hline 15 & (epi)gallocatechin-(epi)catechin & 4.72 & $\mathrm{C}_{30} \mathrm{H}_{26} \mathrm{O}_{13}$ & 593.1301 & 593.1377 & -7.2 & 13.1 & $527.1595,353.0831,191.0279$ \\
\hline 16 & myricetin hexoside isomer & 5.21 & $\mathrm{C}_{21} \mathrm{H}_{20} \mathrm{O}_{13}$ & 479.0831 & 479.0881 & -6.0 & 7.1 & $329.0988,219.0590,173.015$ \\
\hline 17 & 4-hydroxybenzoic acid & 5.34 & $\mathrm{C}_{7} \mathrm{H}_{6} \mathrm{O}_{3}$ & 137.0244 & 137.0297 & -4.7 & 8.9 & 155.0405 \\
\hline 18 & Kaempferol-methoxy-methyl ether & 5.68 & $\mathrm{C}_{17} \mathrm{H}_{14} \mathrm{O}_{7}$ & 329.0878 & 329.0892 & -8.8 & 16.8 & \\
\hline 19 & catechin hexoside & 6.10 & $\mathrm{C}_{21} \mathrm{H}_{24} \mathrm{O}_{11}$ & 451.1246 & 451.1262 & -5.8 & 13 & $365.1499,289.0896$ \\
\hline 20 & (epi)catechin-(epi)catechin & 6.16 & $\mathrm{C}_{30} \mathrm{H}_{24} \mathrm{O}_{12}$ & 575.1195 & 575.1176 & -4.5 & 32.2 & $375.0857,287.0674$ \\
\hline 21 & (epi)gallocatechin-(epi)catechin & 6.45 & $\mathrm{C}_{30} \mathrm{H}_{26} \mathrm{O}_{13}$ & 593.1301 & 593.1386 & -6.2 & 8.5 & 289.0786 \\
\hline 22 & procyanidin trimer & 6.53 & $\mathrm{C}_{45} \mathrm{H}_{38} \mathrm{O}_{20}$ & 897.1884 & 897.1867 & -3.6 & 40.5 & $479.1002,425.0999,289.0788$ \\
\hline 23 & syringic acid isomer & 6.58 & $\mathrm{C}_{9} \mathrm{H}_{10} \mathrm{O}_{5}$ & 197.0455 & 197.0429 & -3.5 & 8.5 & $\begin{array}{l}179.0428,165.0259 \\
0\end{array}$ \\
\hline 24 & (epi)catechin-(epi)catechin & 6.66 & $\mathrm{C}_{30} \mathrm{H}_{26} \mathrm{O}_{12}$ & 577.1351 & 577.1327 & -5.4 & 8.7 & $425.1043,289.0572$ \\
\hline 25 & (epi)azfelechin glucoside derivative & 6.77 & $\mathrm{C}_{27} \mathrm{H}_{30} \mathrm{O}_{16}$ & 609.1402 & 609.1441 & 3.4 & 18.5 & 289.0815 \\
\hline 26 & (epi)gallocatechin & 6.90 & $\mathrm{C}_{15} \mathrm{H}_{14} \mathrm{O}_{7}$ & 305.0667 & 305.0666 & -2.4 & 3.8 & \\
\hline 27 & catechin & 7.15 & $\mathrm{C}_{15} \mathrm{H}_{14} \mathrm{O}_{6}$ & 289.0718 & 289.0721 & -3.6 & 2.2 & \\
\hline 28 & isoquercitrin & 7.27 & $\mathrm{C}_{21} \mathrm{H}_{20} \mathrm{O}_{12}$ & 463.0882 & 463.0841 & -3.3 & 15.2 & $329.0981,289.0823,175.0313$ \\
\hline 29 & (epi)catechin-glucoside & 7.54 & $\mathrm{C}_{21} \mathrm{H}_{24} \mathrm{O}_{11}$ & $\begin{array}{l}450.0002 \\
451.1246\end{array}$ & 451.1293 & $\begin{array}{l}-3.3 \\
-3.6\end{array}$ & 5.7 & $\begin{array}{c}289.0811,229.1064 \\
\text { 28. }\end{array}$ \\
\hline 30 & (epi)catechin-glucoside & 7.60 & $\mathrm{C}_{21} \mathrm{H}_{24} \mathrm{O}_{11}$ & 451.1246 & 451.1284 & -3.5 & 9 & $289.0789,229.106$ \\
\hline 31 & $\begin{array}{l}\text { caffeic acid } \\
\text { chiduc }\end{array}$ & 7.74 & $\mathrm{C}_{9} \mathrm{H}_{8} \mathrm{O}_{4}$ & 179.035 & 179.0311 & -4.4 & 19.4 & 135.0506 \\
\hline 32 & (epi)gallocatechin-(epi)catechin & 7.94 & $\mathrm{C}_{30} \mathrm{H}_{26} \mathrm{O}_{13}$ & 593.1301 & 593.1393 & -3.5 & 17.6 & $407.1109,289.0777$ \\
\hline 33 & (epi)catechin-(epi)catechin & 8.04 & $\mathrm{C}_{30} \mathrm{H}_{26} \mathrm{O}_{12}$ & 577.1351 & 577.1353 & -2.9 & 10.8 & $451.1465,327.1182,289.1005$ \\
\hline 34 & trihydroxy(iso)flavanol-(epi)catechin & 8.76 & $\mathrm{C}_{30} \mathrm{H}_{26} \mathrm{O}_{11}$ & 561.1402 & 561.1471 & -3.1 & 8 & $\begin{array}{l}409.1127,289.1006 \\
\end{array}$ \\
\hline 35 & eriodictyol-O-hexoside & 8.97 & $\mathrm{C}_{21} \mathrm{H}_{22} \mathrm{O}_{11}$ & 449.1242 & 449.1234 & -32.1 & 5.8 & $317.1382,191.0213,179.059$ \\
\hline 36 & luteolin-6-C-glucoside (orientin) & 8.99 & $\mathrm{C}_{21} \mathrm{H}_{20} \mathrm{O}_{11}$ & 447.0933 & 447.0978 & -3.4 & 9.9 & 431.1019, 377.1054 \\
\hline 37 & catechin deoxyhexose & 9.08 & $\mathrm{C}_{21} \mathrm{H}_{24} \mathrm{O}_{10}$ & 435.1297 & 435.123 & -3.6 & 3.3 & $317.1349,289.1005,173.0148$ \\
\hline 38 & catechin deoxyhexose & 9.23 & $\mathrm{C}_{21} \mathrm{H}_{24} \mathrm{O}_{10}$ & 435.1297 & 435.1228 & -3.1 & 6.2 & $317.1156,289.0993$ \\
\hline 39 & (epi)gallocatechin-(epi)catechin & 9.26 & $\mathrm{C}_{30} \mathrm{H}_{26} \mathrm{O}_{13}$ & 593.1301 & 593.1318 & -3.6 & 29.2 & $289.0815,179.0423$ \\
\hline 40 & (epi)gallocatechin-(epi)catechin & 9.39 & $\mathrm{C}_{30} \mathrm{H}_{26} \mathrm{O}_{13}$ & 593.1301 & 593.1398 & -3.3 & 12.9 & $289.0843,179.0432$ \\
\hline 41 & parasorboside & 9.41 & $\mathrm{C}_{12} \mathrm{H}_{20} \mathrm{O}_{8}$ & 291.1085 & 291.1094 & -3.2 & 4.8 & 173.0161 \\
\hline 42 & (epi)fisetinidol & 9.51 & $\mathrm{C}_{15} \mathrm{H}_{14} \mathrm{O}_{5}$ & 273.0768 & 273.0766 & $\begin{array}{c}-5.2 \\
2.1\end{array}$ & $\begin{array}{l}4.0 \\
10.3\end{array}$ & 149.0343 \\
\hline 43 & lucenin-2,4-methyl ether (diosmetin 6,8-di-C-hexoside) & 9.91 & $\mathrm{C}_{28} \mathrm{H}_{32} \mathrm{O}_{16}$ & 623.1618 & 623.1646 & 3.5 & 2.1 & $567.1891,381.1902$ \\
\hline 44 & $\begin{array}{l}\text { (epi)azfelechin glucoside derivative } \\
\text { (epres }\end{array}$ & 10.03 & $\mathrm{C}_{27} \mathrm{H}_{30} \mathrm{O}_{16}$ & 609.1402 & 609.1457 & -3.2 & 12.7 & $435.1368,273.0773$ \\
\hline 45 & $\begin{array}{l}\text { (epi)adigalloylglucose isomer } \\
\text { distive }\end{array}$ & 10.03 & $\mathrm{C}_{20} \mathrm{H}_{20} \mathrm{O}_{14}$ & 483.078 & 483.0931 & $\begin{array}{l}-3.2 \\
-6.6\end{array}$ & 19.6 & $377.1008,321.0748$ \\
\hline 46 & digalloylglucose isomer & 10.22 & $\mathrm{C}_{20} \mathrm{H}_{20} \mathrm{O}_{14}$ & 483.078 & 483.0934 & -6.7 & 15.7 & $377.1008,321.0748$ \\
\hline 47 & eriodictyol-O-hexoside & 10.26 & $\mathrm{C}_{21} \mathrm{H}_{22} \mathrm{O}_{11}$ & 449.1289 & 449.1235 & -3.5 & 10 & 173.0162 \\
\hline 48 & (epi)azfelechin glucoside & 10.38 & $\mathrm{C}_{21} \mathrm{H}_{24} \mathrm{O}_{10}$ & 435.1297 & 435.1237 & -3.2 & 8.7 & 273.0888 \\
\hline 49 & trihydroxy(iso)flavanol-(epi)catechin & 10.78 & $\mathrm{C}_{30} \mathrm{H}_{26} \mathrm{O}_{11}$ & 561.1402 & 561.1479 & -3.6 & 6.5 & $449.1333,289.1656$ \\
\hline 50 & eriodictyol & 11.08 & $\mathrm{C}_{15} \mathrm{H}_{12} \mathrm{O}_{6}$ & 287.0561 & 287.0566 & -3.4 & 9.3 & - \\
\hline 51 & daidzin & 11.18 & $\mathrm{C}_{21} \mathrm{H}_{20} \mathrm{O}_{9}$ & 415.1035 & 415.1068 & -2.2 & 15.8 & $359.0897,281.077$ \\
\hline
\end{tabular}


Table 2. Cont.

\begin{tabular}{|c|c|c|c|c|c|c|c|c|}
\hline Peak N & Compound & RT (min) & Molecular Formula & Calculated Mass $[\mathrm{M}-\mathrm{H}]^{-}$ & Experimental Mass $[\mathrm{M}-\mathrm{H}]^{-}$ & Error (ppm) & mSigma & In Source Fragments \\
\hline 52 & (epi)azfelechin glucoside & 11.23 & $\mathrm{C}_{21} \mathrm{H}_{24} \mathrm{O}_{10}$ & 435.1297 & 435.1236 & -3.9 & 6.9 & 273.0867 \\
\hline 53 & (epi)azfelechin glucoside & 11.32 & $\mathrm{C}_{21} \mathrm{H}_{24} \mathrm{O}_{10}$ & 435.1297 & 435.1233 & -3.3 & 5.6 & 273.1089 \\
\hline 54 & lucenin-2,4-methyl ether (diosmetin 6,8-di-C-hexoside) & 11.40 & $\mathrm{C}_{28} \mathrm{H}_{32} \mathrm{O}_{16}$ & 623.1618 & 623.1641 & 2.3 & 17.5 & $463.1415,435.1502$ \\
\hline 55 & trihydroxy(iso)flavanol-(epi)catechin & 11.59 & $\mathrm{C}_{30} \mathrm{H}_{26} \mathrm{O}_{11}$ & 561.1402 & 561.1467 & -2.4 & 10.1 & 479.0994, 439.1923, 409.1001 \\
\hline 56 & baicalin & 11.69 & $\mathrm{C}_{21} \mathrm{H}_{18} \mathrm{O}_{11}$ & 445.0776 & 445.0716 & -3.3 & 7.2 & $891.1876,291.1229,173.0167$ \\
\hline 57 & trihydroxy(iso)flavanol-(epi)catechin & 11.72 & $\mathrm{C}_{30} \mathrm{H}_{26} \mathrm{O}_{11}$ & 561.1402 & 561.1476 & -3.3 & 27.7 & $483.1149,435.144,313.0921$ \\
\hline 58 & (epi)fisetinidol & 11.85 & $\mathrm{C}_{15} \mathrm{H}_{14} \mathrm{O}_{5}$ & 273.0768 & 273.0768 & -3.3 & 7.8 & $149.0291,123.0508$ \\
\hline 59 & (epi)fisetinidol & 12.00 & $\mathrm{C}_{15} \mathrm{H}_{14} \mathrm{O}_{5}$ & 273.0768 & 273.0763 & -3.6 & 13.4 & $173.0142,149.0276,123.0383$ \\
\hline 60 & (epi)catechin-(epi)catechin & 12.00 & $\mathrm{C}_{30} \mathrm{H}_{26} \mathrm{O}_{12}$ & 577.1351 & 577.1335 & -3.4 & 35.6 & $447.1115,273.0863$ \\
\hline 61 & (epi)azfelechin glucoside derivative & 12.21 & $\mathrm{C}_{27} \mathrm{H}_{30} \mathrm{O}_{16}$ & 609.1402 & 609.1444 & -3.1 & 9.3 & $561.1585,435.137,273.0849$ \\
\hline 62 & isorhamnetin-O-dihexoside & 12.41 & $\mathrm{C}_{28} \mathrm{H}_{32} \mathrm{O}_{17}$ & 639.1203 & 639.1273 & -4.9 & 11.5 & $561.1618,477.1199$ \\
\hline 63 & rosmarinic acid & 12.47 & $\mathrm{C}_{18} \mathrm{H}_{16} \mathrm{O}_{8}$ & 359.0772 & 359.0795 & -4.3 & 18.3 & $289.0587,217.1178$ \\
\hline 64 & (epi)azfelechin glucoside & 12.74 & $\mathrm{C}_{21} \mathrm{H}_{24} \mathrm{O}_{10}$ & 435.1297 & 435.1231 & -3.9 & 7.2 & $\begin{array}{l}273.0879 \\
\text { 2070 }\end{array}$ \\
\hline 65 & (epi)azfelechin glucoside & 12.87 & $\mathrm{C}_{21} \mathrm{H}_{24} \mathrm{O}_{10}$ & 435.1297 & 435.1235 & -3.8 & 5.1 & 273.0877 \\
\hline 66 & trihydroxy(iso)flavanol-(epi)catechin & 12.99 & $\mathrm{C}_{30} \mathrm{H}_{26} \mathrm{O}_{11}$ & 561.1402 & 561.1483 & -3.2 & 18.1 & $345.0787,251.0658$ \\
\hline 67 & trihydroxy(iso)flavanol-(epi)catechin & 13.16 & $\mathrm{C}_{30} \mathrm{H}_{26} \mathrm{O}_{11}$ & 561.1402 & 561.1478 & -3.3 & 16.4 & $449.164,435.1421$ \\
\hline 68 & (epi)azfelechin glucoside & 13.21 & $\mathrm{C}_{21} \mathrm{H}_{24} \mathrm{O}_{10}$ & 435.1297 & 435.1234 & -3.5 & 12.1 & 273.0846 \\
\hline 69 & $\begin{array}{l}\text { syringic acid hexoside } \\
\text { sidis }\end{array}$ & 13.24 & $\mathrm{C}_{15} \mathrm{H}_{20} \mathrm{O}_{10}$ & 359.0984 & 359.0992 & 2.6 & 11.3 & 251.0642 \\
\hline 70 & phloretin dihexoside & 13.60 & $\mathrm{C}_{27} \mathrm{H}_{33} \mathrm{O}_{15}$ & 597.1825 & 597.1818 & -3.3 & 6.3 & $477.1434,387.2049$ \\
\hline 71 & trihydroxy(iso)flavanol-(epi)catechin & 14.03 & $\mathrm{C}_{30} \mathrm{H}_{26} \mathrm{O}_{11}$ & 561.1402 & 561.1481 & -3.8 & 19.6 & $447.1132,413.1034$ \\
\hline 72 & (epi)azfelechin-(epi)azfelechin -(epi)catechin & 14.47 & $\mathrm{C}_{45} \mathrm{H}_{38} \mathrm{O}_{16}$ & 833.2087 & 833.2046 & -3.2 & 14.5 & $\begin{aligned} 561.1625,429.2246,331.1875 \\
179.0626\end{aligned}$ \\
\hline 73 & diosmetin-rutinoside & 14.65 & $\mathrm{C}_{28} \mathrm{H}_{32} \mathrm{O}_{15}$ & 607.1668 & 607.1625 & 7.1 & 13.5 & $\begin{array}{c}1799.066 \\
411.1149,343.0861\end{array}$ \\
\hline 74 & trihydroxyflavanone dimer & 14.67 & $\mathrm{C}_{30} \mathrm{H}_{26} \mathrm{O}_{10}$ & 545.1453 & 545.1426 & -3.7 & 16.1 & 447.1091, 431.2326, 173.0151 \\
\hline 75 & $\begin{array}{l}\text { (epi)catechin-(epi)catechin } \\
\text { (a) }\end{array}$ & 15.47 & $\mathrm{C}_{30} \mathrm{H}_{24} \mathrm{O}_{12}$ & 575.1195 & 575.1188 & -3.5 & 24.9 & $378.1656,285.0525$ \\
\hline 76 & durantin A & 15.54 & $\mathrm{C}_{22} \mathrm{H}_{20} \mathrm{O} 9$ & 427.1035 & 427.1048 & -2.6 & 12.6 & $341.0792,249.0385$ \\
\hline 77 & diosmetin-rutinoside & 16.12 & $\mathrm{C}_{28} \mathrm{H}_{32} \mathrm{O}_{15}$ & 607.1668 & 607.1647 & 3.6 & 17.3 & $467.1121,341.0817$ \\
\hline 78 & 2,6-dihydroxy-3-methyl-4-O-(6"-O-galloyl- $\beta$-D & 16.64 & $\mathrm{C}_{27} \mathrm{H}_{26} \mathrm{O}_{13}$ & 557.1301 & 557.1256 & 7.9 & 16.4 & $521.276,431.2423,269.1844$ \\
\hline 79 & caffeoylglucaric acid derivative & 17.56 & $\mathrm{C}_{24} \mathrm{H}_{52} \mathrm{O}_{14}$ & 563.3284 & 563.3261 & 4.2 & 12.9 & $547.2124,471.2775$ \\
\hline 80 & myricetin derivative & 18.35 & $\mathrm{C}_{30} \mathrm{H}_{60} \mathrm{O}_{20}$ & 739.3605 & 739.363 & -3.4 & 19.8 & $565.3407,489.2849$ \\
\hline 81 & methylsyringin & 18.47 & $\mathrm{C}_{18} \mathrm{H}_{26} \mathrm{O}_{9}$ & 385.1504 & 385.1522 & 2.3 & 11.8 & $295.1442,265.1188$ \\
\hline 82 & (epi)azfelechin glucoside derivative & 19.00 & $\mathrm{C}_{27} \mathrm{H}_{52} \mathrm{O}_{12}$ & 567.3386 & 567.3376 & -3.4 & 2.2 & 475.3059 \\
\hline
\end{tabular}


3.2. Free and Bound Phenolic Compounds Content and Their In Vitro Antioxidant and Hypoglycemic Activity

The present study quantified free (FPC) and bound (BPC) phenolic compounds in banana passion fruit, following the HPLC-ESI-TOF-MS analyses. In Table 3, the absolute and relative content levels of all free phenolic compounds identified are reported.

Table 3. Quantification of free phenolic compounds (FPC) of banana passion fruit pulp $\left(\mathrm{mg} 100 \mathrm{~g}^{-1} \mathrm{~d} . \mathrm{m}\right.$.).

\begin{tabular}{|c|c|c|c|}
\hline \multirow{2}{*}{ Peak N } & \multirow{2}{*}{ Compound } & FPC & \multirow{2}{*}{$\%$ of the total FPC } \\
\hline & & (mg $100 \mathrm{~g}^{-1}$ d.m.) & \\
\hline \multicolumn{4}{|c|}{ Hydroxybenzoic acid derivatives } \\
\hline 81 & methylsyringin & $361.51 \pm 0.89$ & 24.6 \\
\hline \multicolumn{4}{|c|}{ Hydroxycinnamic acid derivatives } \\
\hline 3 & dicaffeoylquinic acid & $144.95 \pm 0.63$ & 9.9 \\
\hline 4 & dicaffeoylquinic acid & $86.63 \pm 0.27$ & 5.9 \\
\hline 6 & dicaffeoylquinic acid & $43.64 \pm 0.51$ & 3.0 \\
\hline 7 & caffeoyltartaric acid derivative & $93.08 \pm 0.67$ & 6.3 \\
\hline 79 & caffeoylglucaric acid derivative & $48.39 \pm 0.29$ & 3.3 \\
\hline \multicolumn{4}{|c|}{ Flavonols and flavone derivatives } \\
\hline 16 & myricetin hexoside isomer & $2.08 \pm 0.21$ & 0.1 \\
\hline 18 & kaempferol-methoxy-methyl ether & $0.67 \pm 0.09$ & 0.05 \\
\hline 28 & isoquercitrin & $0.98 \pm 0.01$ & 0.1 \\
\hline 36 & eriodictyol-O-hexoside & $3.67 \pm 0.10$ & 0.2 \\
\hline 43 & lucenin-2,4-methyl ether (diosmetin 6,8-di-C-hexoside) & $1.09 \pm 0.05$ & 0.1 \\
\hline 47 & eriodictyol-O-hexoside & $1.46 \pm 0.02$ & 0.1 \\
\hline 50 & eriodictyol & $1.72 \pm 0.02$ & 0.1 \\
\hline 54 & lucenin-2,4-methyl ether (diosmetin 6,8-di-C-hexoside) & $0.56 \pm 0.01$ & 0.04 \\
\hline 56 & baicalin & $1.60 \pm 0.03$ & 0.1 \\
\hline 62 & isorhamnetin-O-dihexoside & $1.43 \pm 0.12$ & 0.1 \\
\hline 70 & naringenin hexose derivate & $2.03 \pm 0.23$ & 0.1 \\
\hline 74 & trihydroxyflavanone dimer & $0.90 \pm 0.01$ & 0.1 \\
\hline 80 & myricetin derivative & $5.09 \pm 0.16$ & 0.3 \\
\hline \multicolumn{4}{|c|}{ Flavan-3-ol derivatives } \\
\hline 5 & (epi)gallocatechin & $5.66 \pm 0.11$ & 0.4 \\
\hline 8 & (epi)azfelechin glucoside derivative & $105.20 \pm 1.38$ & 7.2 \\
\hline 11 & (epi)gallocatechin & $3.46 \pm 0.64$ & 0.2 \\
\hline 12 & (epi)gallocatechin & $24.81 \pm 0.60$ & 1.7 \\
\hline 13 & (epi)gallocatechin & $26.34 \pm 0.41$ & 1.8 \\
\hline 14 & (epi)gallocatechin & $1.42 \pm 0.09$ & 0.1 \\
\hline 19 & catechin hexoside & $2.44 \pm 0.19$ & 0.2 \\
\hline 27 & catechin & $9.61 \pm 0.53$ & 0.7 \\
\hline 29 & (epi)catechin-glucoside & $2.74 \pm 0.11$ & 0.2 \\
\hline 30 & (epi)catechin-glucoside & $5.66 \pm 0.08$ & 0.4 \\
\hline 37 & catechin deoxyhexose & $7.26 \pm 0.37$ & 0.5 \\
\hline 38 & catechin deoxyhexose & $33.42 \pm 0.70$ & 2.3 \\
\hline 44 & (epi)azfelechin glucoside derivative & $3.36 \pm 0.05$ & 0.2 \\
\hline 48 & (epi)azfelechin glucoside & $52.28 \pm 0.99$ & 3.6 \\
\hline 52 & (epi)azfelechin glucoside & $8.08 \pm 0.30$ & 0.6 \\
\hline 53 & (epi)azfelechin glucoside & $2.90 \pm 0.06$ & 0.2 \\
\hline 58 & (epi)fisetinidol & $76.74 \pm 1.03$ & 5.2 \\
\hline 59 & (epi)fisetinidol & $2.96 \pm 0.44$ & 0.2 \\
\hline 61 & (epi)azfelechin glucoside derivative & $1.33 \pm 0.01$ & 0.1 \\
\hline 64 & (epi)azfelechin glucoside & $6.02 \pm 0.20$ & 0.4 \\
\hline 65 & (epi)azfelechin glucoside & $38.56 \pm 1.06$ & 2.6 \\
\hline 68 & (epi)azfelechin glucoside & $9.36 \pm 0.14$ & 0.6 \\
\hline 82 & (epi)azfelechin glucoside derivative & $91.18 \pm 1.51$ & 6.2 \\
\hline 15 & (epi)gallocatechin-(epi)catechin & $64.45 \pm 2.02$ & 4.4 \\
\hline 21 & (epi)gallocatechin-(epi)catechin & $3.58 \pm 0.07$ & 0.2 \\
\hline 22 & procyanidin trimer & $1.29 \pm 0.03$ & 0.1 \\
\hline 24 & (epi)-catechin-(epi)catechin & $10.20 \pm 0.80$ & 0.7 \\
\hline 33 & (epi)-catechin-(epi)catechin & $2.53 \pm 0.05$ & 0.2 \\
\hline 34 & trihydroxy(iso)flavanol-(epi)catechin & $5.95 \pm 0.27$ & 0.4 \\
\hline 49 & trihydroxy(iso)flavanol-(epi)catechin & $45.48 \pm 0.39$ & 3.1 \\
\hline 55 & trihydroxy(iso)flavanol-(epi)catechin & $4.92 \pm 0.08$ & 0.3 \\
\hline 67 & trihydroxy(iso)flavanol-(epi)catechin & $3.94 \pm 0.10$ & 0.3 \\
\hline 72 & (epi)azfelechin-(epi)azfelechin-(epi)catechin & $3.57 \pm 0.04$ & 0.2 \\
\hline
\end{tabular}

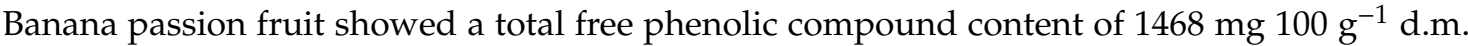
(dry matter).

Bound phenolic compound content is reported in Table 4. The total BPC amount was $878 \mathrm{mg}$ $100 \mathrm{~g}^{-1} \mathrm{~d} . \mathrm{m}$. The sum of 3,4-dihydroxybenzoic acid and syringic acid hexoside represented $75.7 \%$ of the total bound fraction. 
Table 4. Quantification of bound phenolic compounds (BPC) of banana passion fruit pulp (mg $100 \mathrm{~g}^{-1}$ d.m.).

\begin{tabular}{|c|c|c|c|}
\hline \multirow{2}{*}{ Peak N } & \multirow{2}{*}{ Compound } & BPC & \multirow{2}{*}{$\%$ of the total BPC } \\
\hline & & (mg $100 \mathrm{~g}^{-1}$ d.m.) & \\
\hline \multicolumn{4}{|c|}{ Hydroxybenzoic acid derivatives } \\
\hline 9 & 3,4-dihydroxybenzoic acid & $327.62 \pm 2.29$ & 37.3 \\
\hline 10 & syringic acid & $60.65 \pm 1.13$ & 6.9 \\
\hline 17 & 4-hydroxybenzoic acid & $7.63 \pm 1.07$ & 0.9 \\
\hline 23 & syringic acid & $61.68 \pm 1.39$ & 7.0 \\
\hline 69 & syringic acid hexoside & $337.57 \pm 2.98$ & 38.4 \\
\hline \multicolumn{4}{|c|}{ Hydroxicinnamic acid derivatives } \\
\hline 31 & caffeic acid & $1.38 \pm 0.06$ & 0.2 \\
\hline 45 & digalloylglucose isomer & $6.16 \pm 0.14$ & 0.7 \\
\hline 46 & digalloylglucose isomer & $10.25 \pm 0.02$ & 1.2 \\
\hline 63 & rosmarinic acid & $4.84 \pm 0.08$ & 0.6 \\
\hline \multicolumn{4}{|c|}{ Flavonol and flavone derivatives } \\
\hline 36 & luteolin-6-C-glucoside (orientin) & $3.09 \pm 0.07$ & 0.4 \\
\hline 51 & daidzin & $0.54 \pm 0.02$ & 0.1 \\
\hline 73 & diosmetin-7-O-rutinoside & $0.57 \pm 0.02$ & 0.1 \\
\hline 76 & durantin A & $0.44 \pm 0.06$ & 0.1 \\
\hline 77 & diosmetin-7-O-rutinoside & $1.09 \pm 0.03$ & 0.1 \\
\hline \multicolumn{4}{|c|}{ Flavan-3-ol derivatives } \\
\hline 12 & (epi)gallocatechin & $7.21 \pm 0.18$ & 0.8 \\
\hline 13 & (epi)gallocatechin & $3.30 \pm 0.10$ & 0.4 \\
\hline 25 & (epi)azfelechin glucoside derivative & $1.32 \pm 0.05$ & 0.2 \\
\hline 26 & (epi)gallocatechin & $1.07 \pm 0.01$ & 0.1 \\
\hline 27 & catechin & $9.88 \pm 0.40$ & 1.1 \\
\hline 42 & (epi)fisetinidol & $7.17 \pm 0.27$ & 0.8 \\
\hline 48 & (epi)azfelechin glucoside & $2.63 \pm 0.19$ & 0.3 \\
\hline 59 & (epi)fisetinidol & $1.97 \pm 0.11$ & 0.2 \\
\hline 64 & (epi)azfelechin glucoside & $1.35 \pm 0.07$ & 0.2 \\
\hline 65 & (epi)azfelechin glucoside & $2.50 \pm 0.01$ & 0.3 \\
\hline 68 & (epi)azfelechin glucoside & $3.35 \pm 0.13$ & 0.4 \\
\hline 20 & (epi)catechin-(epi)catechin & $1.85 \pm 0.02$ & 0.2 \\
\hline 32 & (epi)gallocatechin-(epi)catechin & $0.29 \pm 0.01$ & 0.03 \\
\hline 39 & (epi)gallocatechin-(epi)catechin & $2.05 \pm 0.08$ & 0.2 \\
\hline 40 & (epi)gallocatechin-(epi)catechin & $3.03 \pm 0.04$ & 0.3 \\
\hline 57 & trihydroxy(iso)flavanol-(epi)catechin & $0.85 \pm 0.09$ & 0.1 \\
\hline 60 & (epi)-catechin-(epi)catechin & $1.89 \pm 0.18$ & 0.2 \\
\hline 66 & trihydroxy(iso)flavanol-(epi)catechin & $1.34 \pm 0.06$ & 0.2 \\
\hline 71 & trihydroxy(iso)flavanol-(epi)catechin & $1.19 \pm 0.12$ & 0.1 \\
\hline 75 & (epi)catechin-(epi)catechin & $0.73 \pm 0.05$ & 0.1 \\
\hline
\end{tabular}

Briefly, the total phenolic content (sum of free and bound phenolic fraction) was $2356 \mathrm{mg}$ $100 \mathrm{~g}^{-1}$ d.m., as reported in Figure 1. FPC represented the most abundant fraction of compounds, amounting to $62.3 \%$ of the total content, whereas BPC constituted $33.7 \%$ of the total phenolic compounds. These results are in agreement with previous studies that found that phenolics in fruits were mainly present in soluble free form, at approximately $62 \%-96 \%$, and only around $24 \%$ of total phenolics belonged to BPC [42]. Similar percentages were previously observed for banana and palm oil fruits, with $33.1 \%$ and $33.2 \%$ BPC in the total phenolic content, respectively [43].

The in vitro antioxidant capacities of free and bound phenolic extracts of banana passion fruit, evaluated by three different assays, are shown in Figure 2.

IC50 values of $\alpha$-amylase inhibition were $20.8 \pm 0.9$ and $61.9 \pm 1.2 \mu \mathrm{g} / \mathrm{mL}$ in FPC and BPC extracts, respectively. 


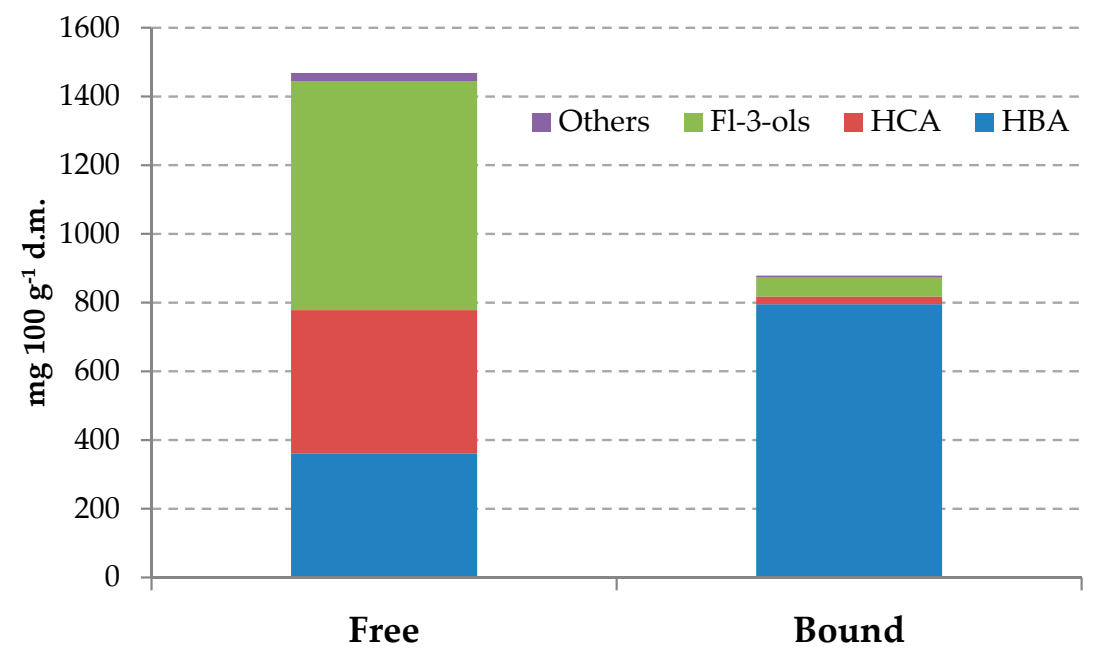

Figure 1. Distribution of phenolic compound classes in free and bound fraction. HBA, hydroxybenzoic acid derivatives; HCA, hydroxycinnamic acid derivatives; Fl-3-ols, flavan-3-ol derivatives.
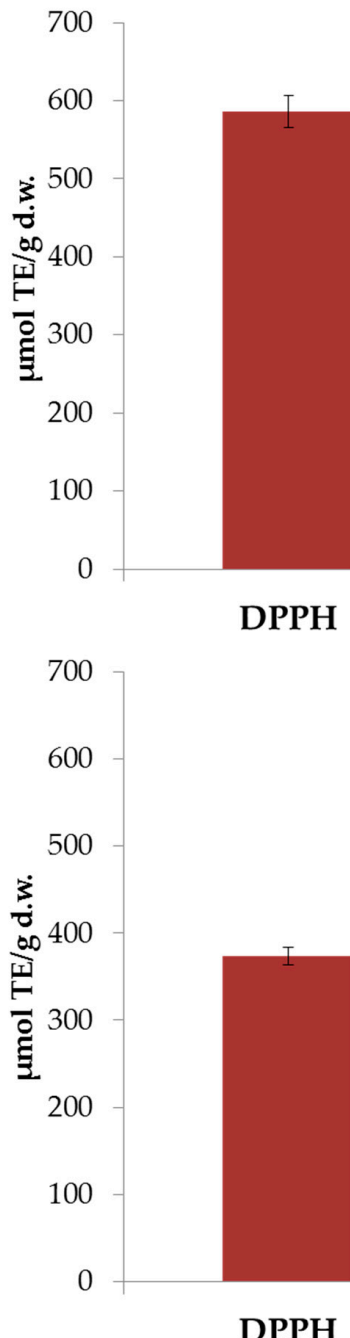

A

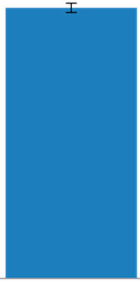

FRAP

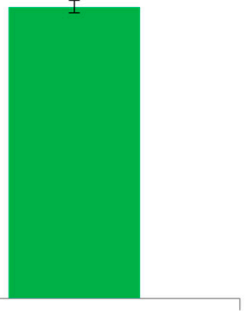

ABTS

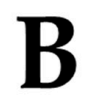

B

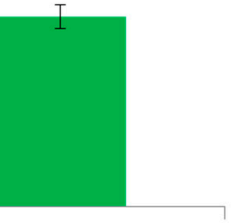

ABTS

Figure 2. In vitro antioxidant capacities of free (A) and bound (B) phenolic compounds of banana passion fruit pulp. 


\section{Discussion}

In the literature, several works have quantified the phenolic content of Passiflora species, with a particular focus on Passiflora edulis (passion fruit) and tropical fruits in general. Most of these works have determined the total phenolic, total flavonoid and total anthocyanin contents by spectrophotometric techniques. Septembre-Malaterre et al. [29] reported passion fruit as the richest source of phenolic compounds, compared with litchi, banana, pineapple, mango and papaya. Da Silva et al. [44] found $34.8 \mathrm{mg} \mathrm{kg}^{-1} \mathrm{~d} . \mathrm{m}$. of total anthocyanins and $603.7 \mathrm{mg} \mathrm{kg}^{-1} \mathrm{~d} . \mathrm{m}$. of total yellow flavonoids in passion fruit pulp, and similar values were also reported for its by-products; moreover, they determined a total phenolic content of $7651 \mathrm{mg}$ gallic acid equivalent (GAE) $\mathrm{kg}^{-1} \mathrm{~d}$.m., defining passion fruit as a good source of these compounds. A similar trend was reported by Ruiz-Torralba and co-workers [24], who noticed that Passiflora edulis f. flavicarpa fruit had the highest phenolic content of 52 fruits sold in Spain. Brat et al. [45], with the objective of creating a French database of the polyphenolic content of fruits and vegetables, reported $718 \mathrm{mg}$ of $\mathrm{GAE} \mathrm{kg}{ }^{-1}$ fresh matter (f.m.) for passion fruit, and, considering a mean moisture value of $80 \%$, this content could be expressed as $3590 \mathrm{mg}$ of GAE $\mathrm{kg}^{-1}$ d.m. Similar values were also found by Vasco et al. [3] for passion fruit, whereas much higher contents were detected for banana passion fruit, which, with 10,100 mg of GAE kg-1 f.m., was included as a "high level phenolic compounds" fruit, together with Andean blackberry and Capulí cherry peel. Again, Contreras-Calderón et al. [16] found for banana passion fruit the highest phenolic content, with a total of $6350 \mathrm{mg}$ of GAE kg-1 f.m. In the same work, another banana passion fruit of the genus Passiflora tarminiana was taken into account, which was found to be even richer in phenolics $(10,180 \mathrm{mg}$ of GAE kg ${ }^{-1}$ f.m.). However, they analyzed pulp and seeds together, and it is important to highlight that seeds contain higher amounts of phenolic compounds than pulp.

Hydroxycinnamic acids and their derivatives constituted $28.4 \%$ of the total amount of FPC; in particular, dicaffeoylquinic acid derivatives were the main compounds (275 $\mathrm{mg} 100 \mathrm{~g}^{-1} \mathrm{~d}$.m.). Meinhart et al. [46] investigated the content of dicaffeoylquinic acids in 64 fruits consumed in Brazil, showing that passion fruit presented the highest content of 3,5-dicaffeoylquinic acid, reaching $312 \mathrm{mg} \mathrm{kg}^{-1} \mathrm{~d} . \mathrm{m}$. in one sample. Other authors [26] found $14 \mathrm{mg}$ of 3,5-dicaffeoylquinic acid per $100 \mathrm{~mL}$ of passion fruit juice.

The total quantity of flavan-3-ols in the FPC extract was $667 \mathrm{mg} 100 \mathrm{~g}^{-1} \mathrm{~d} . \mathrm{m}$. The most abundant compound was an isomer of (epi)azfelechin glucoside, followed by the monomer unit (epi)fisetinidol. These findings agree with García-Ruiz et al. [15], who indicated flavan-3-ols as the major constituents of the phenolic fraction of banana passion fruit pulp, after freeze-drying; the authors indicated (epi)azfelechin and its glucoside derivatives as the most abundant compounds among this class of phenolics.

FPC and BPC were mainly composed by phenolic acids (53\% and $93.1 \%$ of the total amount, respectively). Flavan-3-ols were mainly present in the FPC extract, representing 45.4\% of total FPC; contrarily, they contributed only $6.3 \%$ of the bound phenolic fraction. Very low amounts were observed for flavonols and flavone derivatives, representing $1.3 \%$ and $1.1 \%$ of FPC and BPC, respectively.

The antioxidant capacity of free phenolic compounds (Figure 2A) measured by the DPPH assay was $587 \mu \mathrm{mol} \mathrm{TE} / \mathrm{g}$ (d.w.) of the sample. This result strongly agrees with that reported by Garcia-Ruiz et al. [15]. A lower value was found by Ruiz-Torralba and co-workers [24]. However, as reported by Garcia-Ruiz et al. [15], variability from 0.9 to $608 \mu \mathrm{mol} \mathrm{TE} / \mathrm{g}$ (d.w.) was previously obtained in banana passion fruit. The antioxidant capacity tested by the FRAP assay was $346.2 \mu \mathrm{mol} \mathrm{TE} / \mathrm{g}$ (d.w.); this value is in the same order of magnitude as that obtained by Contreras-Calderón et al. [16] and higher than that obtained by Ruiz-Torralba et al. [24]. Finally, the ABTS assay reported an antioxidant capacity of $373.1 \mu \mathrm{mol} \mathrm{TE} / \mathrm{g}$ (d.w.); this value agrees with that obtained by Contreras-Calderón et al. [16], while a higher value was found by Simirgiotis and co-workers [12]. Generally, the present data confirmed that the antioxidant capacity of banana passion fruit is higher than that obtained in other fruits [15,16,24,47]. In fact, according to Garcia-Ruiz et al. [15], banana passion fruit reported an antioxidant capacity, measured by $\mathrm{DPPH}$, that was more than 10 times higher than that 
reported for Physalis peruviana and Carica papaya. Comparing our data with the results reported by Ruiz-Torralba et al. [24] for other tropical fruits, banana passion fruit showed DPPH values from 11 to 66 times higher than starfruit, cherimoya, mango, maracuya and pitaya; similar results were also found for the ABTS (2.5-17.6 times higher in banana passion fruit) and FRAP (4.7-39.4 times higher in banana passion fruit) assays. ABTS and DPPH values which were more than 300 times lower than banana passion fruit were described by Beserra-Almeida et al. [47] for Brazilian fruits such as jackfruit, mangaba, murici, papaya, pineapple, sapodilla, soursop, sweetsop, tamarind and umbu.

Figure $2 \mathrm{~B}$ shows the antioxidant capacities of the bound phenolic extracts. As expected, the values obtained for bound phenolic extracts were lower than those measured for free phenolics. The DPPH and ABTS values obtained in this work were 6.7 and 2.2 times higher than those obtained in araticum fruit pulp [48]. Moreover, the DPPH and FRAP results were higher than those reported for papaya fruit [49].

Pearson's correlations between phenolic content and antioxidant capacity assays are reported in Table 5.

Table 5. Correlation coefficients $(p<0.001)$ obtained between phenolic content and antioxidant assays in banana passion fruit.

\begin{tabular}{cccc}
\hline Compounds & DPPH & ABTS & FRAP \\
\hline Total phenolic compounds & 0.9987 & 0.9902 & 0.9835 \\
HBA & -0.9913 & -0.9961 & -0.9925 \\
HCA & 0.9930 & 0.9965 & 0.9938 \\
Flavan-3-ols & 0.9931 & 0.9966 & 0.9936 \\
Others & 0.9969 & 0.9963 & 0.9919 \\
\hline
\end{tabular}

As reported in Table 5, several positive correlations in the range of 0.9835-0.9987 were found. These data agreed with the results described by other authors [15,47]. Negative correlations were shown between hydroxybenzoic derivatives and antioxidant capacity assays. Positive correlations were also noticed between the different antioxidant capacity assays $(r=0.9903, p<0.001$ between DPPH and ABTS; $r=0.9847, p<0.001$ between DPPH and FRAP; $r=0.9914, p<0.001$ between ABTS and FRAP), confirming the suitability of the three assays to measure the antioxidant capacity in this fruit. Similar results were also noticed by other authors [16,47].

Inhibition analysis of $\alpha$-amylase activity was conducted in order to corroborate the potential antihyperglycemic activity of phenolic compounds contained in passiflora passion fruit extracts. As reported by several authors [50,51], the inhibition of some key digestive enzymes, such as $\alpha$-amylase, is a good strategy to treat or inhibit hyperglycemia. The highest $\alpha$-amylase inhibition shown by the FPC extract could be justified by the higher content of phenolic compounds compared to the BPC extract. Moreover, the FPC extract contains high amounts of flavonoids that seem to be particularly involved in the hypoglycemic process [18]. Besides this, high correlations were found between $\alpha$-amylase inhibition and total phenolic content $(r=0.9948, p<0.001)$ and between $\alpha$-amylase inhibition and total flavonoid content $(r=0.9999, p<0.001)$. Data obtained for the FPC extract was very close to that obtained by Shanmugam et al. [52,53] in Passiflora leschenaultia and Passiflora subpeltata fruits. Higher values were found by Loizzo et al. [18] for free phenolic extracts of banana passion fruit and other Passiflora fruits; however, this discrepancy could be due to botanical or agronomical factors, and the different extraction methods for phenolic compounds.

\section{Conclusions}

This paper represents a further contribution to the investigation of the phytochemical composition of banana passion fruit, a lesser explored species found in Ecuador and other Latin American countries.

HPLC-ESI-TOF-MS was successfully applied in order to identify and quantify free and bound phenolic compounds extracted from banana passion fruit (Passiflora mollissima) pulp. By means of 
this analytical technique, 82 compounds were tentatively identified, and 80 of them were quantified. Free phenolic compounds represented the most abundant fraction of phenolics. The total phenolic acids (hydroxybenzoic plus hydroxycinnamic acid derivatives) were the highest concentrated phenolic group in both free and bound phenolic fractions. However, flavan-3-ols were the most concentrated compounds of the free phenolic fraction, considering the single classes. Moreover, the antioxidant capacities measured with three different assays showed that banana passion fruit presented a high antioxidant activity, significantly higher than in other fruits. These new data regarding free and bound phenolic identification and quantification and the evaluation of the antioxidant capacity of banana passion fruit may represent an incentive to promote the use of this fruit in food preparation. Indeed, this new information about banana passion fruit's phenolic composition could also be used to characterize banana passion fruits from different countries and/or different food processes. Briefly, the high phenolic content and the antioxidant and antihyperglycemic capacity found in this fruit confirmed its potential as a functional fruit. However, in vitro assays are only explorative determinations; because of this, further in vivo analyses are necessary in order to confirm these data and to corroborate the bioaccessibility and bioavailability of the phenolic compounds present in banana passion fruit.

Supplementary Materials: The following are available online at http://www.mdpi.com/2076-3921/9/7/628/s1, Figure S1: Base peak chromatogram of free (FPC) and bound (BPC) phenolic compounds in banana passion fruit obtained by HPLC-ESI-TOF-MS.

Author Contributions: Conceptualization, V.V. and A.M.G.-C.; methodology, V.V. and A.M.G.-C.; formal analysis, A.R.-T., E.G. and J.G.F.-H.; resources, J.G.F.-H.; data curation, E.G.; V.V.; writing-original draft preparation, E.G.; writing-review and editing, A.M.G.-C., E.J.G.-H., B.G.-V. and V.V.; supervision, V.V. All authors have read and agreed to the published version of the manuscript.

Funding: This research received no external funding

Acknowledgments: Vito Verardo thanks the Spanish Ministry of Economy and Competitiveness (MINECO) for "Ramon y Cajal" contract (RYC-2015-18795). Elisa Giambanelli thanks the Spanish Ministry of Economy and Competitiveness (MINECO) for "Juan de la Cierva" contract (FJCI-2017-33216). Arancha Ruíz Torralba thanks the Ministerio de Educación y Formación Profesional for the FPU fellowship (FPU16/02536).

Conflicts of Interest: The authors declare no conflict of interest.

\section{References}

1. Egea, M.B.; Pereira-Netto, A.B. Bioactive compound-rich, virtually unknown, edible fruits from the Atlantic Rainforest: Changes in antioxidant activity and related bioactive compounds during ripening. Eur. Food Res. Technol. 2019, 245, 1081-1093. [CrossRef]

2. Schmidt, H.D.O.; Rockett, F.C.; Pagno, C.H.; Possa, J.; Assis, R.Q.; De Oliveira, V.R.; Da Silva, V.L.; Flôres, S.H. Vitamin and bioactive compound diversity of seven fruit species from south Brazil. J. Sci. Food Agric. 2019, 99, 3307-3317. [CrossRef] [PubMed]

3. Vasco, C.; Ruales, J.; Kamal-Eldin, A. Total phenolic compounds and antioxidant capacities of major fruits from Ecuador. Food Chem. 2008, 111, 816-823. [CrossRef]

4. Galluzzi, G.; López Noriega, I. Conservation and use of genetic resources of underutilized crops in the Americas-A continental analysis. Sustainability 2014, 6, 980-1017. [CrossRef]

5. Carrillo-Perdomo, E.; Aller, A.; Cruz-Quintana, S.M.; Giampieri, F.; Alvarez-Suarez, J.M. Andean berries from Ecuador: A review on botany, agronomy, chemistry and health potential. J. Berry Res. 2015, 5, 49-69. [CrossRef]

6. Tene, V.; Malagón, O.; Finzi, P.V.; Vidari, G.; Armijos, C.; Zaragoza, T. An ethnobotanical survey of medicinal plants used in Loja and Zammora-Chichipe, Ecuador. J. Ethnopharmacol. 2006, 111, 63-81. [CrossRef] [PubMed]

7. Dhawan, K.; Dhawan, S.; Sharma, A. Passiflora: A review update. J. Ethnopharmacol. 2004, 94, 1-23. [CrossRef] 
8. Deng, J.; Zhou, Y.; Bai, M.; Li, H.; Li, L. Anxiolytic and sedative activities of Passiflora edulis f. activities and flavonoid compositions of Passiflora edulis "edulis" and Passiflora edulis "flavicarpa". J. Ethnopharmacol. 2010, 133, 1085-1090.

9. Kris-Etherton, P.M.; Hecker, K.D.; Bonanome, A.B.; Coval, S.M.; Binkoski, A.E.; Hilpert, K.F.; Griel, A.E.; Etherton, T.D. Bioactive compounds in foods: Their role in the prevention of cardiovascular disease and cancer. Am. J. Med. 2002, 113, 71-88. [CrossRef]

10. Ángel-Coca, C.; Nates-Parra, G.; Ospina-Torres, R.; Melo Ortiz, C.D.; Amaya Márquez, M. Floral and reproductive biology of the gulupa Passiflora edulis Sims F. edulis. Caldasia 2011, 2, 433-451.

11. Ballesteros-Vivas, D.; Alvarez-Rivera, G.; Ibánez, E.; Parada-Alfonso, F.; Cifuentes, A. Integrated strategy for the extraction and profiling of bioactive metabolites from Passiflora mollissima seeds combining pressurized-liquid extraction and gas/liquid chromatography-high resolution mass spectrometry. J. Chromatogr. A 2019, 1595, 144-157. [CrossRef] [PubMed]

12. Simirgiotis, M.; Schmeda-Hirschmann, G.; Bórquez, J.; Kennelly, E.J. The Passiflora tripartita (Banana Passion) fruit: A source of bioactive flavonoid C-glycosides isolated by HSCCC and characterized by HPLC-DAD-ESI/MS/MS. Molecules 2013, 18, 1672-1692. [CrossRef] [PubMed]

13. Zucolotto, S.M.; Fagundes, C.; Reginatto, F.H.; Ramos, F.A.; Castellanos, L.; Duque, C.; Schenkel, E.P. Analysis of C-glycosyl flavonoide from South American Passiflora species by HPLC-DAD and HPLC-MS. Phytochem. Analysis 2011, 23, 232-239.

14. Aguillón-Osma, J.; Luzardo-Ocampo, I.; Cuellar-Nuňez, M.L.; Maldonado-Celis, M.E.; Loango-Chamorro, N.; Campos-Vega, R. Impact of in vitro gastrointestinal digestion on the bioaccessibility and antioxidant capacity of bioactive compounds from Passion fruit (Passiflora edulis) leaves and juice extracts. J. Food Biochem. 2019, 43, e12879. [CrossRef]

15. Garcïa-Ruiz, A.; Girones-Vilaplana, A.; León, P.; Moreno, D.A.; Stinco, C.M.; Meléndez-Martínez, A.J.; Ruales, J. Banana passion fruit (Passiflora mollissima (Kunth) L.H. Bayley): Microencapsulation, phytochemical composition and antioxidant capacity. Molecules 2017, 22, 85. [CrossRef] [PubMed]

16. Contreras-Calderón, J.; Calderón-Jaimes, L.; Guerra-Hernández, E.; García-Villanova, B. Antioxidant capacity, phenolic content and vitamin $\mathrm{C}$ in pulp, peel and seed from 24 exotic fruits from Colombia. Food Res. Int. 2011, 44, 2047-2053. [CrossRef]

17. Gowd, V.; Karim, N.; Shishir, M.R.I.; Xie, L.; Chen, W. Dietary polyphenols to combat the metabolic diseases via altering gut microbiota. Trends Food Sci. Tech. 2019, 93, 81-93. [CrossRef]

18. Loizzo, M.R.; Lucci, P.; Núñez, O.; Tundis, R.; Balzano, M.; Frega, N.G.; Conte, L.; Moret, S.; Filatova, D.; Moyano, E.; et al. Native colombian fruits and their by-products: Phenolic profile, antioxidant activity and hypoglycaemic potential. Foods 2019, 8, 89. [CrossRef]

19. Carmona-Hernandez, J.C.; Taborda-Ocampo, G.; Valdez, J.C.; Bolling, B.W.; González-Correa, C.H. polyphenol extracts from three colombian passifloras (passion fruits) prevent inflammation-induced barrier dysfunction of caco-2 cells. Molecules 2019, 24, 4614. [CrossRef]

20. Acosta-Estrada, B.; Gutiérrez-Uribe, J.A.; Serna-Saldívar, S.O. Bound phenolics in foods, a review. Food Chem. 2014, 152, 46-55. [CrossRef]

21. Chandrasekara, A.; Shahidi, F. Content of insoluble bound phenolics in millets and their contribution to antioxidant capacity. J. Agric. Food Chem. 2010, 58, 6706-6714. [CrossRef] [PubMed]

22. AOAC International. Official Methods of Analysis of AOAC International, 18th ed.; AOAC Intl.: Gaithersburg, MD, USA, 2007.

23. Gómez-Caravaca, A.; López-Cobo, A.; Verardo, V.; Segura-Carretero, A.; Fernández-Gutiérrez, A. HPLC-DAD-q-TOF-MS as a powerful platform for the determination of phenolic and other polar compounds in the edible part of mango and its by-products (Peel, Seed, and Seed Husk). Electrophoresis 2016, 37, 1072-1084. [CrossRef] [PubMed]

24. Ruiz Torralba, A.; Guerra Hernández, E.; García-Villanova Ruiz, B. Antioxidant capacity, polyphenol content and contribution to dietary intake of 52 fruits sold in Spain. CYTA J. Food 2018, 16, 1131-1138. [CrossRef]

25. Leporini, M.; Loizzo, M.R.; Sicari, V.; Pellicanò, T.M.; Reitano, A.; Dugay, A.; Deguin, B.; Tundis, R. Citrus $\times$ clementina hort. juice enriched with its by-products (Peels and Leaves): Chemical composition, in vitro bioactivity, and impact of processing. Antioxidants 2020, 9, 298. [CrossRef] [PubMed] 
26. Spínola, V.; Pinto, J.; Castilho, P.C. Identification and quantification of phenolic compounds of selected fruits from Madeira Island by HPLC-DAD-ESI-MS and screening for their antioxidant activity. Food Chem. 2015, 173, 14-30. [CrossRef] [PubMed]

27. Cuvelier, M.E.; Richard, H.; Berset, C. Antioxidative activity and phenolic composition of pilot-plant and commercial extracts of sage and rosemary. J. Am. Oil Chem. Soc. 1996, 73, 645-652. [CrossRef]

28. Espin, S.; Gonzalez-Manzano, S.; Taco, V.; Poveda, C.; Ayuda-Durán, B.; Gonzalez-Paramas, A.M.; Santos-Buelga, C. Phenolic composition and antioxidant capacity of yellow and purple-red Ecuadorian cultivars of tree tomato (Solanum betaceum Cav.). Food Chem. 2016, 194, 1073-1080. [CrossRef]

29. Septembre-Malaterre, A.; Stanislas, G.; Douraguia, E.; Gonthier, M.P. Evaluation of nutritional and antioxidant properties of the tropical fruits banana, litchi, mango, papaya, passion fruit and pineapple cultivated in Réunion French Island. Food Chem. 2016, 212, 225-233. [CrossRef]

30. Díaz de Cerio, E.; Gómez-Caravaca, A.M.; Verardo, V.; Fernández-Gutiérrez, A.; Segura-Carretero, A. Determination of guava (Psidium guajava L.) leaf phenolic compounds using HPLC-DAD-QTOF-MS. J. Funct. Foods 2016, 22, 376-388. [CrossRef]

31. Guimarães, R.; Barros, L.; Dueñas, M.; Carvalho, A.M.; Queiroz, M.J.R.P.; Santos-Buelga, C.; Ferreira, I.C.F.R. Characterisation of phenolic compounds in wild fruits from Northeastern Portugal. Food Chem. 2013, 141, 3721-3730. [CrossRef]

32. Pereira, O.R.; Peres, A.M.; Silva, A.M.S.; Domingues, M.R.M.; Cardoso, S.M. Simultaneous characterization and quantification of phenolic compounds in Thymus $x$ citriodorus using a validated HPLC-UV and ESI-MS combined method. Food Res. Int. 2013, 54, 1773-1780. [CrossRef]

33. Anton, D.; Matt, D.; Pedastsaar, P.; Bender, I.; Kazimierczak, R.; Roasto, M.; Kaart, T.; Luik, A.; Püssa, T. Three-year comparative study of polyphenol contents and antioxidant capacities in fruits of tomato (Lycopersicon esculentum Mill.) cultivars grown under organic and conventional conditions. J. Agric. Food Chem. 2014, 62, 5173-5180. [CrossRef] [PubMed]

34. Domínguez-Rodríguez, G.; Concepción García, M.; Plaza, M.; Marina, M.L. Revalorization of Passiflora species peels as a sustainable source of antioxidant phenolic compounds. Sci. Total Environ. 2019, 696, 134030. [CrossRef]

35. Ahmad, N.; Zeb, F.; Ahmad, I.; Wang, F. Repenins A-D, four new antioxidative coumarinolignoids from Duranta repens Linn. Bioorg. Med. Chem. Lett. 2009, 19, 3521-3524. [CrossRef] [PubMed]

36. Li, J.; Wang, Y.H.; Smillie, T.J.; Khan, I.A. Identification of phenolic compounds from Scutellaria lateriflora by liquid chromatography with ultraviolet photodiode array and electrospray ionization tandem mass spectrometry. J. Pharmaceut. Biomed. 2012, 63, 120-127. [CrossRef] [PubMed]

37. Shu, J.; Chou, G.; Wang, Z. Two new benzophenone glycosides from the fruit of Psidium guajava L. Fitoterapia 2010, 81, 532-535. [CrossRef]

38. De Souza Mesquita, L.M.; Caria, C.R.E.P.; Santos, P.S.; Ruy, C.C.; Da Silva Lima, N.; Moreira, D.K.T.; Da Rocha, C.Q.; Murador, D.C.; De Rosso, V.V.; Gambero, A.; et al. Modulatory effect of polyphenolic compounds from the mangrove tree Rhizophora mangle L. on non-alcoholic fatty liver disease and insulin resistance in high-fat diet obese mice. Molecules 2018, 23, E2114. [CrossRef]

39. Song, Y.; Wei, X.Q.; Li, M.Y.; Duan, X.W.; Sun, Y.M.; Yang, R.L.; Su, X.D.; Huang, R.M.; Wang, H. Nutritional composition and antioxidant properties of the fruits of a chinese wild passiflora foetida. Molecules 2018, 23, 459. [CrossRef]

40. Verardo, V.; Arráez-Román, D.; Segura-Carretero, A.; Marconi, E.; Fernández-Gutiérrez, A.; Caboni, M.F. Identification of buckwheat phenolic compounds by reverse phase high performance liquid chromatography-electrospray ionization-time of flight-mass spectrometry (RP-HPLC-ESI-TOF-MS). J. Cereal Sci. 2010, 52, 170-176. [CrossRef]

41. AbadGarcía, B.; Berrueta, L.A.; Garmón-Lobato, S.; Gallo, B.; Vicente, F. A general analytical strategy for the characterization of phenolic compounds in fruit juices by high-performance liquid chromatography with diode array detection coupled to electrospary ionization and triple quadrupole mass spectrometry. J. Chromatogr. A 2009, 1216, 5398-55415. [CrossRef]

42. Sun, J.; Chu, Y.F.; Wu, X.; Liu, R.H. Antioxidant and antiproliferative activities of common fruits. J. Agric. Food Chem. 2002, 50, 7449-7454. [CrossRef]

43. Neo, Y.P.; Ariffin, A.; Tan, C.P.; Tan, Y.A. Phenolic acid analysis and antioxidant activity assessment of oil palm (E. Guineensis) fruit extracts. Food Chem. 2010, 122, 353-359. [CrossRef] 
44. Da Silva, L.M.R.; De Figueiredo, E.A.T.; Ricardo, N.M.P.S.; Vieira, I.G.P.; De Figueiredo, R.W.; Brasil, I.M.; Gomes, C.L. Quantification of bioactive compounds in pulps and by-products of tropical fruits from Brazil. Food Chem. 2014, 143, 398-404. [CrossRef] [PubMed]

45. Brat, P.; George, S.; Bellamy, A.; DuChaffaut, L.; Scalbert, A.; Mennen, L.; Arnault, N.; Amiot, M.J. Daily polyphenol intake in France from fruit and vegetables. J. Nutr. 2006, 136, 2368-2373. [CrossRef] [PubMed]

46. Meinhart, A.D.; Damin, F.M.; Caldeirão, L.; De Jesus Filho, M.; Da Silva, L.C.; Da Silca Constant, L.; Teixera Filho, J.; Wagner, R.; Teixera Godoy, H. Chlorogenic and caffeic acids in 64 fruits consumed in Brazil. Food Chem. 2019, 286, 51-63. [CrossRef] [PubMed]

47. Beserra Almeida, M.M.; Machado de Sousa, P.H.; Campos Arriaga, A.M.; Matias do Prado, G.; De Carvalho Magalhães, C.E.; Arraes Maia, G.; Gomes de Lemos, T.L. Bioactive compounds and antioxidant activity of fresh exotic fruits from northeastern Brazil. Food Res. Int. 2011, 44, 2155-2159. [CrossRef]

48. Arruda, H.S.; Araujo Pereira, G.; Rodrigues de Morais, D.; Nogueira Eberlin, M.; Pastore, G.M. Determination of free, esterified, glycosylated and insoluble-bound phenolics composition in the edible part of araticum fruit (Annona crassiflora Mart.) and its by-products by HPLC-ESI-MS/MS. Food Chem. 2018, 245, 738-749. [CrossRef] [PubMed]

49. Uribe, E.; Delgadillo, A.; Giovagnoli-Vicuña, C.; Quispe-Fuentes, I.; Zura-Bravo, L. Extraction techniques for bioactive compounds and antioxidant capacity determination of chilean papaya (Vasconcellea pubescens) Fruit. J. Chem. 2015, 2015, 347532. [CrossRef]

50. Wu, L.; Liu, Y.; Qin, Y.; Wang, L.; Wu, Z. HPLC-ESI-qTOF-MS/MS characterization, antioxidant activities and inhibitory ability of digestive enzymes with molecular docking analysis of various parts of raspberry (Rubus ideaus L.). Antioxidants 2019, 8, 274. [CrossRef] [PubMed]

51. Balli, D.; Cecchi, L.; Khatib, M.; Bellumori, M.; Cairone, F.; Carradori, S.; Zengin, G.; Cesa, S.; Innocenti, M.; Mulinacci, N. Characterization of arils juice and peel decoction of fifteen varieties of Punica granatum L.: A Focus on anthocyanins, ellagitannins and polysaccharides. Antioxidants 2020, 9, 238. [CrossRef] [PubMed]

52. Shanmugam, S.; Dutra Sandes, R.D.; Rajan, M.; Santos Leite Neta, M.T.; Dos Santos Lima, B.; Melo de Jesus, M.J.; Denadai, M.; Narain, N.; Thangaraj, P.; Russo Serafini, M.; et al. Volatile profiling and UHPLC-QqQ-MS/MS polyphenol analysis of Passiflora leschenaultii DC. fruits and its anti-radical and anti-diabetic properties. Food Res. Int. 2020, 133, 109202. [CrossRef] [PubMed]

53. Shanmugam, S.; Alcântara Gomes, I.; Denadai, M.; Dos Santos Lima, B.; De Souza Araújo, A.A.; Narain, N.; Santos Leite Neta, M.T.; Russo Serafini, M.; Quintans-Júnior, L.J.; Thangaraj, P. UHPLC-QqQ-MS/MS identification, quantification of polyphenols from Passiflora subpeltata fruit pulp and determination of nutritional, antioxidant, $\alpha$-amylase and $\alpha$-glucosidase key enzymes inhibition properties. Food Res. Int. 2018, 108, 611-620. [CrossRef] [PubMed]

(C) 2020 by the authors. Licensee MDPI, Basel, Switzerland. This article is an open access article distributed under the terms and conditions of the Creative Commons Attribution (CC BY) license (http://creativecommons.org/licenses/by/4.0/). 